\title{
Dynamics at the angle of repose: jamming, bistability, and collapse
}

\author{
J M Luck† and Anita Mehta \\ † Service de Physique Théoriqueł, CEA Saclay, 91191 Gif-sur-Yvette cedex, France \\ $\ddagger$ S N Bose National Centre for Basic Sciences, Block JD, Sector 3, Salt Lake, \\ Calcutta 700098, India
}

\begin{abstract}
When a sandpile relaxes under vibration, it is known that its measured angle of repose is bistable in a range of values bounded by a material-dependent maximal angle of stability; thus, at the same angle of repose, a sandpile can be stationary or avalanching, depending on its history. In the nearly jammed slow dynamical regime, sandpile collapse to a zero angle of repose can also occur, as a rare event. We claim here that fluctuations of dilatancy (or local density) are the key ingredient that can explain such varied phenomena. In this work, we model the dynamics of the angle of repose and of the density fluctuations, in the presence of external noise, by means of coupled stochastic equations. Among other things, we are able to describe sandpile collapse in terms of an activated process, where an effective temperature (related to the density as well as to the external vibration intensity) competes against the configurational barriers created by the density fluctuations.
\end{abstract}

PACS numbers: 45.70.-n, 61.43.Gt, 89.75.Fb, 05.65.+b, 05.40.-a

E-mail: luck@spht.saclay.cea.fr, anita@bose.res.in 


\section{Introduction}

The angle of repose [1] in a sandpile has been a recurrent enigma for physicists, both in paradigmatic and in realistic terms. Ideas of self-organised criticality [2] used the angle of repose as a paradigm - it was rather appealing to think that sandpiles relaxed to a marginally stable state with a unique critical angle, which resulted from a universal and extensive dynamics. Interest in the angle of repose only increased when it became obvious to most physicists that this picture was too simplistic [3]; in fact the angle of repose is multiply-valued within a certain range, and relaxational behaviour about it reveals a great deal about intrinsic length scales within the sandpile [4.

We first summarise the relevant phenomenology. Sandpiles formed by the deposition of grains on surfaces have sides that are typically inclined at a finite angle to the horizontal $\S$. This is the angle of repose $\theta_{\mathrm{r}}$ : in practice what is seen is that it can take a range of values before spontaneous flow occurs, i.e., the sandpile becomes unstable to further deposition. The limiting value of the angle before avalanching occurs is known as the maximal angle of stability $\theta_{\mathrm{m}}$. The difference between these two angles, which is characteristic of a given material [1], is often referred to as the Bagnold angle [5]. Also, sandpiles show strong hysteresis $\|$ : thus, depending on its conditions of formation, a sandpile can either be stable or in motion at any angle $\theta$ such that $\theta_{\mathrm{r}}<\theta<\theta_{\mathrm{m}}$. This bistable behaviour has been studied theoretically and experimentally [6, 7], but its dynamical origin has not been clearly explained so far, to the best of our knowledge.

Another enigma concerns the dynamics around the angle of repose. Early experiments in the physics literature [8] indicated that, when subjected to a large vibration intensity, the angle of repose of a sandpile would decay to zero logarithmically with time: gently vibrated sandpiles, on the other hand, after an initial logarithmic decay, remained 'jammed' at a finite angle of repose for experimentally observable times. This led, in a theory [4] that followed, to the suggestion that different mechanisms were responsible for decay in the two dynamical regimes. In the strongly vibrated sandpile, grains relaxed independently of each other, and the angle of repose decayed logarithmically to zero; for gently vibrated sandpiles, there was insufficient inertial energy given to individual grains, and collective dynamics were responsible for the 'jamming' observed at finite angles. The mean-field approach adopted in that work was adequate to identify the different rates of decay; a treatment of the fluctuations, needed for example to explain bistability or jamming at the angle of repose, was, however, beyond its scope.

In this work, our objectives are, firstly, to propose a possible mechanism for observed bistable behaviour; secondly, and equally importantly, to construct a coherent picture,

$\S$ Sandpiles formed by other processes, e.g. by drainage through a narrow pore within a flat box, also have angles of repose which depend on their history; their description can also be included, with minor modifications, in our formulation.

\| This is a result of the 'athermal' nature of sandpiles, i.e., a consequence of the fact that grain sizes are too large for the ambient temperature to have any effect on their dynamics. Thus configurations that would be dissolved away by Brownian motion in liquids or gases, survive in sandpiles. 
involving both fast and slow dynamical modes, of relaxation at the angle of repose. The present work is based on coupled stochastic equations which are similar, but not identical, to those proposed earlier in 4]. The exact analytical treatment to these equations will provide, in the slow dynamical regime of interest, a description of the dual problems of stability and collapse of the angle of repose.

We now summarise the main results of the present work. Our basic picture is that fluctuations of local density (especially when they occur coherently, in the limit of a small perturbation) are the collective excitations responsible for stabilising the angle of repose, and for giving it its characteristic width, known as the Bagnold angle [5]. Density fluctuations can occur because of shape effects [9] or friction [1, 10]; they are the manifestation in our model of dilatancy, first observed by Reynolds [1] in 1885 .

Our theory below essentially relates the Bagnold angle to the Reynolds' dilatancy. In the absence of dilatancy, we suggest that the angle of repose in a vibrated sandpile would decay swiftly to zero 9 . When dilatancy is present, density fluctuations add to the value of the 'bare' angle of repose. In our theory, 'out-of-equilibrium' density fluctuations (such as those that might be found at the start of a shaking process) generate the maximal angle of stability $\theta_{m}$; on the other hand, asymptotic values of density fluctuations 'equilibrated' by vibrations at long times, give rise to the the 'typical' angle of repose $\theta_{\mathrm{r}}$. The Bagnold angle, which is defined [5] as the difference

$$
\delta \theta_{\mathrm{B}}=\theta_{\mathrm{m}}-\theta_{\mathrm{r}},
$$

is thus found to be the difference between nonequilibrium and equilibrium values of the dilatancy for a given material.

Consider a sandpile that has relaxed to $\theta_{\mathrm{r}}$ in the presence of low noise; it is now stabilised by an equilibrium value of the density fluctuations. We ask the question: what is the probability, under these circumstances, that the sandpile collapses to a zero angle of repose? The configurational landscape we are dealing with is that of grains rather close to jamming; density fluctuations involve small (typically intra-cage) displacements of grains about their 'equilibrium' positions in this very disordered network. Typical configurational barrier heights for collapse under such circumstances would be rather high, as they would involve a global rearrangement of grains. This is rather reminiscent of the situation close to the 'dynamical transition' in an earlier random graph model [12] of granular compaction; in both cases, long-range correlations (corresponding to systemwide density fluctuations) need to develop for an appropriate collapse to occur ${ }^{+}$. Clearly such events would be rare; while they would clearly be facilitated by an increase in the external noise, the effect of dilatancy merits more discussion. An increase of dilatancy means that density fluctuations are greater; the effect of disorder is greater and configurational landscapes, rougher. Since sandpile collapse requires global

I In liquids, for instance, the absence of dilatancy is the reason why liquid surfaces do not spontaneously sustain themselves at a non-zero angle to the horizontal.

+ In the case of compaction, 'collapse' corresponds to the attainment of the dynamical transition via a collapse of excess void space; here it corresponds to the collapse of the sandpile to a zero angle of repose. 
rearrangement of grains, it will be less probable in rough (strongly dilatant) landscapes. Our analytical results confirm these qualitative observations; we find spontaneous collapse to be an activated process, with an effective temperature $\varepsilon$ that depends on the ratio of the external noise to the ambient value of the density fluctuations.

\section{The model}

The dynamics of the angle of repose $\theta(t)$ and of the density fluctuations $\phi(t)$ are described by the following coupled stochastic equations:

$$
\begin{aligned}
& \dot{\theta}=-a \theta+b \phi^{2}+\Delta_{1} \eta_{1}(t), \\
& \dot{\phi}=-c \phi+\Delta_{2} \eta_{2}(t) .
\end{aligned}
$$

The parameters $a, \ldots, \Delta_{2}$ are phenomenological constants, while $\eta_{1}(t), \eta_{2}(t)$ are two independent white noises such that

$$
\left\langle\eta_{i}(t) \eta_{j}\left(t^{\prime}\right)\right\rangle=2 \delta_{i j} \delta\left(t-t^{\prime}\right)
$$

Similar equations, which involve spatial rather than temporal coordinates, have been written to describe the orientational structure of bridges in granular media; these have been extensively analysed in concurrent work [13] and their results are in good agreement with independent simulations. Density fluctuations are key to both phenomena; the angle of repose is stabilised by dilatancy, while linear bridges grow at the expense of local density fluctuations.

From a technical viewpoint, the linear equation (2.2) for $\phi(t)$ is known as an Ornstein-Uhlenbeck equation [14, 15], whereas equation (2.1) for $\theta(t)$ is non-linear, as it contains a quadratic coupling to the Ornstein-Uhlenbeck variable $\phi(t)$. In spite of this nonlinearity, we have been able to find the equilibrium state of the coupled equations (2.1), (2.2) analytically - one of the very rare instances where this is possible. In the Appendix, we show that the stationary Fokker-Planck equation describing this equilibrium state can be solved in closed form by means of a quadratic Ansatz (A.13). This ansatz only works for the very special kind of non-linearity here, where (2.1) involves the square of the Ornstein-Uhlenbeck variable $\phi$. We have come across a similar quadratic Ansatz for a problem with a quadratic coupling between two stochastic variables [16].

We now motivate our equations physically. The first terms in (2.1) and (2.2) are suggested on stability grounds: neither the angle of repose nor the dilatancy are allowed to be arbitrarily large for a stable system. The second term in (2.1) affirms that dilatancy underlies the phenomenon of the angle of repose; in the absence of noise, density fluctuations constitute this angle*. The noise in (2.1) represents external vibration, while that in (2.2) is a version of the Edwards compactivity [17, related as it is to purely density-driven effects. In earlier work [4, these were related via fluctuationdissipation relations to effective temperatures for (decoupled) fast and slow dynamics. In

* We write a term proportional to $\phi^{2}$ on symmetry grounds - we would expect it to depend on the magnitude rather than the sign of density fluctuations. 
this work, the inclusion of correlations will be seen to lead to an effective temperature $\varepsilon$, related now to the ratio of these two noises, in the slow dynamical regime.

When the material is weakly dilatant $(c \gg a)$, so that density fluctuations decay quickly to zero (and hence can be neglected), the angle of repose $\theta(t)$ itself obeys an Ornstein-Uhlenbeck equation. It relaxes exponentially fast to an equilibrium state, whose variance

$$
\theta_{\mathrm{eq}}^{2}=\frac{\Delta_{1}^{2}}{a}
$$

is just the zero-dilatancy variance of $\theta$.

The opposite limit where $c \ll a$ is of much greater interest. Here, density fluctuations are long-lived. When, additionally, $\Delta_{1}$ is small, the angle of repose has a slow dynamics reflective of the slowly evolving density fluctuations. These conditions are written more precisely as

$$
\gamma \ll 1, \quad \varepsilon \ll 1,
$$

in terms of two dimensionless parameters:

$$
\gamma=\frac{c}{a}, \quad \varepsilon=\frac{a c^{2} \Delta_{1}^{2}}{b^{2} \Delta_{2}^{4}}=\frac{\theta_{\mathrm{eq}}^{2}}{\theta_{\mathrm{r}}^{2}} .
$$

The second equality follows from (3.6). We see from this that $\varepsilon$ is essentially the ratio between the fluctuations of the angle of repose $\theta$, in the respective limits when it is decoupled from, and coupled to, the density fluctuations $\phi$. Giving $\varepsilon$ the interpretation of an effective temperature (see below), we define the regime (2.5) as a low-temperature and strongly dilatant regime, governed by the slow evolution of density fluctuations.

\section{Dynamics in and out of equilibrium}

Suppose that a sandpile is created in regime (2.5) with very large initial values for the angle $\theta_{0}$ and dilatancy $\phi_{0}$. The initial stage of the dynamics is a transient one; here, the noises are negligible, so that the decay is entirely given by the deterministic parts of (2.1) and (2.2):

$$
\begin{aligned}
& \theta(t)=\left(\theta_{0}-\theta_{\mathrm{m}}\right) \mathrm{e}^{-a t}+\theta_{\mathrm{m}} \mathrm{e}^{-2 c t}, \\
& \phi(t)=\phi_{0} \mathrm{e}^{-c t},
\end{aligned}
$$

with

$$
\theta_{\mathrm{m}} \approx \frac{b \phi_{0}^{2}}{a} .
$$

Thus, density fluctuations $\phi(t)$ relax exponentially, while the trajectory $\theta(t)$ has two separate modes of relaxation:

- a fast (inertial) decay in $\theta(t) \approx \theta_{0} \mathrm{e}^{-a t}$, until $\theta(t)$ is of the order of $\theta_{\mathrm{m}}$,

- a slow (collective) decay in $\theta(t) \approx \theta_{\mathrm{m}} \mathrm{e}^{-2 c t}$.

When $\phi(t)$ and $\theta(t)$ are small enough [i.e., $\phi(t) \sim \phi_{\mathrm{eq}}$ and $\theta(t) \sim \theta_{\mathrm{r}}$, cf. (3.4) and (3.6)] for the noises to have an appreciable effect, the above analysis is no longer 
valid. The system then reaches the equilibrium state of the full non-linear stochastic process represented by (2.1) and (2.2). An exact analytical investigation of this, for all values of the parameters $\gamma$ and $\varepsilon$, is presented in the Appendix.

In order to get a feeling for the more qualitative features of the equilibrium state, we note first that $\phi(t)$ is an Ornstein-Uhlenbeck process with equilibrium variance:

$$
\phi_{\mathrm{eq}}^{2}=\frac{\Delta_{2}^{2}}{c} .
$$

We see next that to a good approximation, the angle $\theta$ adapts instantaneously to the dynamics of $\phi(t)$ in regime (2.5):

$$
\theta(t) \approx \frac{b \phi(t)^{2}}{a}
$$

The two above statements together imply that the distribution of the angle $\theta(t)$ is approximately that of the square of a Gaussian variable. The typically observed angle of repose $\theta_{\mathrm{r}}$ is the time-averaged value

$$
\theta_{\mathrm{r}}=\langle\theta\rangle_{\mathrm{eq}}=\frac{b \phi_{\mathrm{eq}}^{2}}{a}=\frac{b \Delta_{2}^{2}}{a c} .
$$

Equation (3.5) then reads

$$
\theta(t) \approx \theta_{\mathrm{r}} \frac{\phi(t)^{2}}{\phi_{\mathrm{eq}}^{2}} .
$$

Equation (3.7) makes the physics behind the multivalued and history-dependent nature of the angle of repose (referred to in the Introduction) rather clear. Its instantaneous value depends directly on the instantaneous value of the dilatancy; its maximal (stable) value $\theta_{\mathrm{m}}$ is noise-independent $[\mathrm{cf} .(3.3)]$ and depends only on the maximal value of dilatancy that a given material can sustain stably . Sandpiles constructed above this will first decay quickly to it; they will then decay more slowly to a 'typical' angle of repose $\theta_{\mathrm{r}}$. The ratio of these angles is given by

$$
\frac{\theta_{\mathrm{m}}}{\theta_{\mathrm{r}}}=\frac{\phi_{0}^{2}}{\phi_{\mathrm{eq}}^{2}},
$$

so that $\theta_{\mathrm{m}} \gg \theta_{\mathrm{r}}$ for $\phi_{0} \gg \phi_{\text {eq }}$. Since spontaneous flow always occurs above $\theta_{\mathrm{m}}$, it is known as the angle of maximal stability [1].

Below this, i.e., for $\theta_{\mathrm{r}}<\theta<\theta_{\mathrm{m}}$, we have a region of bistability which depends strongly on sandpile history. The above analysis shows that:

- Sandpiles submitted to low noise are stable in this range of angles, at least for long times $\sim 1 / c$.

- Sandpiles submitted to high noise [such that the effects of dilatancy become negligible in (2.1)] continue to decay rapidly in this range of angles, becoming nearly horizontal at short times $\sim 1 / a$.

This provides rather satisfying agreement with earlier work [6] on tilted sandpiles. In that work, model sandpiles were submitted to tilts of varying magnitudes before $\sharp$ Here stability is defined for times of order $1 / c$, assumed to be extremely long. 
being restored to their original (horizontal) state. Large tilting resulted in avalanching, while small tilting did not, even when the post-tilt state involved the same angle of repose. (The reason for this involved the very different effects in each case on granular configurations in the uppermost layers of the sandpile [6].) Bistability at the angle of repose was thus clearly manifest.

Our conclusions are that bistability at the angle of repose is a natural consequence of applied noise (tilt [6] or vibration) in granular systems; a sandpile can either be at rest, or in motion, at the same angle of repose, depending on its history.

\section{The dynamics of sandpile collapse}

We now examine the probability that under the prolonged effect of low noise, the sandpile collapses; thus we look for events where the angle $\theta(t)$ vanishes. Such an event is expected to be very rare in the regime (2.5); in fact it occurs only if the noise $\eta_{1}(t)$ in (2.1) is sufficiently negative for sufficiently long to compensate for the strictly positive term $b \phi^{2}$. The fully analytical confirmation of this argument is presented in the Appendix. It predicts that the equilibrium probability for $\theta$ to be negative:

$$
\Pi=\operatorname{Prob}(\theta<0),
$$

scales throughout regime $(2.5)$ as

$$
\Pi \approx \frac{(2 \varepsilon)^{1 / 4}}{\Gamma(1 / 4)} \mathcal{F}(\zeta), \quad \zeta=\frac{\gamma}{\varepsilon^{1 / 2}}=\frac{b \Delta_{2}^{2}}{a^{3 / 2} \Delta_{1}}
$$

[see (A.33)]. The scaling function $\mathcal{F}(\zeta)$, given explicitly in (A.35), decays monotonically from $\mathcal{F}(0)=1$ to $\mathcal{F}(\infty)=0$ (see Figure 1 ).

We explore further the regime $\zeta \gg 1$, where the 'temperature' $\varepsilon$ is much lower than the 'barrier height' $\gamma^{2}$. Here, the result (A.36) implies that the equilibrium probability of collapse vanishes exponentially fast:

$$
\Pi \sim \exp \left(-\frac{3}{2}\left(\frac{\gamma^{2}}{\varepsilon}\right)^{1 / 3}\right) .
$$

Thus complete collapse becomes an activated process: collapse events occur at Poissonian times, with an exponentially large characteristic time given by an Arrhenius law:

$$
\tau \sim 1 / \Pi \sim \exp \left(\frac{3}{2}\left(\frac{\gamma^{2}}{\varepsilon}\right)^{1 / 3}\right) .
$$

The stretched exponential in (4.3) is intriguing, as it involves a fractional power of the usual barrier-height-to-temperature ratio $\gamma^{2} / \varepsilon$, and therefore raises questions about the nature of the barriers involved. We investigate this further below.

The equilibrium probability for the magnitude $|\phi(t)|$ of the Ornstein-Uhlenbeck process to remain smaller than some $\phi_{1} \ll \phi_{\text {eq }}$ during a time interval $T$, is known to fall off exponentially as

$$
p_{\phi_{1}}(T) \sim \exp \left(-\frac{\pi^{2}}{4} \frac{\phi_{\mathrm{eq}}^{2}}{\phi_{1}^{2}} c T\right) .
$$




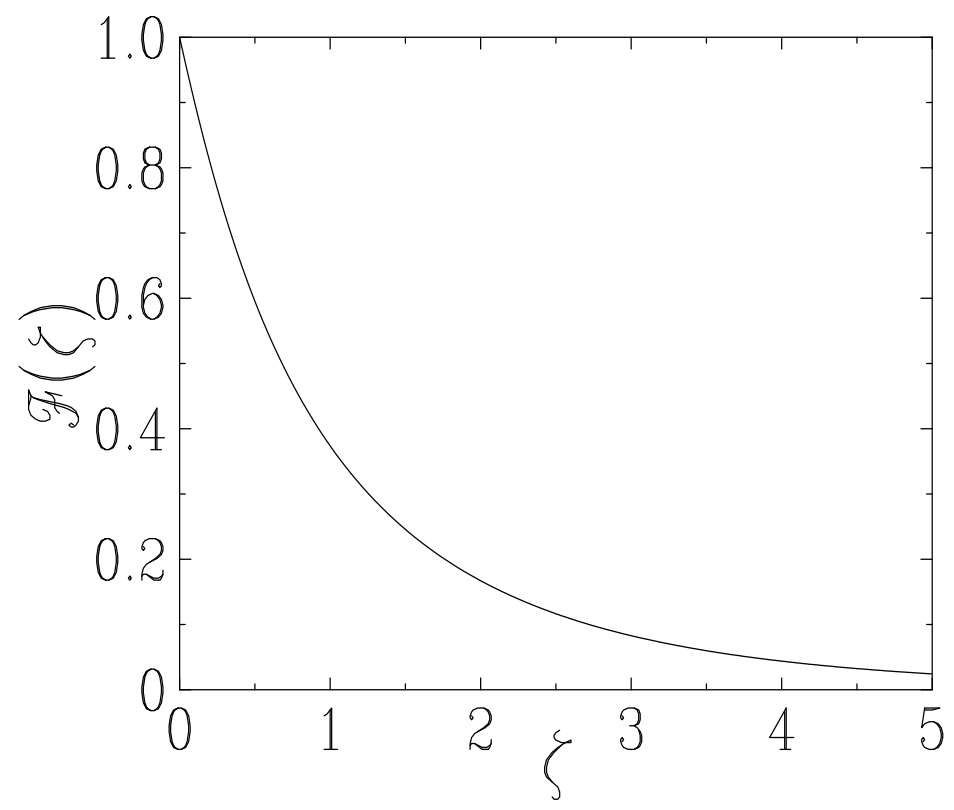

Figure 1. Plot of the function $\mathcal{F}(\zeta)$ entering the scaling law (4.2) of the probability $\Pi$ in the regime (2.5).

We now ask the question: what is the zero-temperature $(\varepsilon=0)$ equilibrium probability $P_{0}(\theta)$ for the angle of repose to assume a very small value $\theta \ll \theta_{\mathrm{r}}$ ? Inserting $\phi_{\text {eq }}^{2} / \phi_{1}^{2} \sim \theta_{\mathrm{r}} / \theta$ [cf. (3.7)] in the estimate (4.5), with $T \sim 1 / a$ (the relaxation time of the uncoupled $\theta$ dynamics), we find that this probability is exponentially small:

$$
P_{0}(\theta) \sim \exp \left(-k \frac{\gamma \theta_{\mathrm{r}}}{\theta}\right) .
$$

This somewhat heuristic argument is borne out by the rigorous analysis of the Appendix, which leads to $k=1$, and also predicts the prefactor [see (A.30)].

In the presence of a low noise intensity $(\varepsilon \ll 1)$, the exponential tail (4.6) gets convoluted with a narrow Gaussian generated by the noise, whose variance is $\theta_{\mathrm{eq}}^{2}=\varepsilon \theta_{\mathrm{r}}^{2}$. This gives

$$
P(\theta) \sim \int_{0}^{\infty} \exp \left(-\frac{\gamma \theta_{\mathrm{r}}}{\theta_{1}}-\frac{\left(\theta-\theta_{1}\right)^{2}}{2 \varepsilon \theta_{\mathrm{r}}^{2}}\right) \mathrm{d} \theta_{1}
$$

Setting $\theta=0$, we get

$$
\Pi \sim \int_{0}^{\infty} \exp \left(-\frac{\gamma \theta_{\mathrm{r}}}{\theta_{1}}-\frac{\theta_{1}^{2}}{2 \varepsilon \theta_{\mathrm{r}}^{2}}\right) \mathrm{d} \theta_{1} .
$$

The saddle-point of the above integral,

$$
\theta_{1} \approx(\gamma \varepsilon)^{1 / 3} \theta_{\mathrm{r}}
$$

leads to the stretched exponential probability distribution (4.3).

The above suggests a very strong analogy with the famous problem of random trapping 18. Consider a particle performing Brownian motion in one dimension, with 
diffusion constant $D$, amidst a concentration $c$ of Poisson-distributed traps; once a trap is reached, the particle ceases to exist. The survival probability $S(t)$ of the particle is therefore the probability that it has not encountered a trap until time $t$. Assuming a uniform distribution of starting points, the fall off of this probability can be estimated by first computing the probability of finding a large region of length $L$ without traps, and then weighing this with the probability that a Brownian particle survives within it for a long time $t$ :

$$
S(t) \sim \int_{0}^{\infty} \exp \left(-c L-\frac{\pi^{2} D t}{L^{2}}\right) \mathrm{d} L
$$

The first exponential factor $\exp (-c L)$ is the probability that a region of length $L$ is free of traps, whereas the second exponential factor is the asymptotic survival probability of a Brownian particle in such a region $\dagger$. The integral is dominated by a saddle-point at

$$
L \approx\left(\frac{2 \pi^{2} D t}{c}\right)^{1 / 3}
$$

whence we recover the well-known estimate

$$
S(t) \sim \exp \left(-\frac{3}{2}\left(2 \pi^{2} c^{2} D t\right)^{1 / 3}\right) .
$$

We now elucidate as fully as possible the nature of the analogy, in order to get further physical insight into the problem of sandpile collapse. Table 1 shows the quantitative correspondence between parameters in both situations. Both the survival probability $S(t)$ and the collapse probability $\Pi$ obey the stretched exponential laws (4.12) and (4.3), with an identical exponent $1 / 3$. Both these anomalous dynamical laws are the result of a saddle-point approximation, which represents in each case an optimisation procedure. In the trapping problem, large regions without traps are improbable, whereas the particle would decay too fast in small ones (it would get absorbed at a boundary); the best compromise (4.11) is found to scale as $L \sim t^{1 / 3}$. In the sandpile problem, angles too far below $\theta_{\mathrm{r}}$ are hard to find, as dilatancy would resist their existence [cf. [4.6)]; on the other hand, angles that are too large would inhibit collapse, given their larger configurational barriers in the face of the noise. Once again, the best compromise (4.9) scales as $\theta_{1} \sim \varepsilon^{1 / 3}$.

We use this analogy to develop the following picture for sandpile collapse. Imagine that the collapse is visualised as the motion of an effective particle (an exciton, say), represented by the collective co-ordinate $\theta(t)$. Under the influence of a temperature $\varepsilon$ the exciton diffuses across a rough landscape defined by the $\phi$ excitations; we can consider this landscape to be a frozen background, since the decay rate of $\theta$ is much faster than that of $\phi$. Valleys are separated by $\phi$ barriers whose typical height scales as $\gamma$; however, sandpile collapse actually involves the traversal of a much lower optimal barrier, given by (4.3). The process of sandpile collapse can therefore be visualised as the $\theta$ exciton's $\dagger \dagger$ This probability is $\exp \left(-D q^{2} t\right)$, with $q=\pi / L$ coming from the Dirichlet boundary conditions at the absorbing endpoints. 


\begin{tabular}{|l|c|c|}
\hline Problem & trapping & sandpile collapse \\
\hline Physical quantity & $S(t)$ & $\Pi$ \\
Large parameter & $2 \pi^{2} D t$ & $1 / \varepsilon$ \\
Control parameter & $c$ & $\gamma$ \\
\hline Integration variable & $L$ & $\theta_{\mathrm{r}} / \theta_{1}$ \\
Saddle-point value & $\left(2 \pi^{2} D t / c\right)^{1 / 3}$ & $(\gamma \varepsilon)^{-1 / 3}$ \\
\hline Result & $(4.12)$ & $(4.3)$ \\
\hline
\end{tabular}

Table 1. Quantitative correspondence between the derivations of the stretched exponential laws (4.12) and (4.3) in the one-dimensional random trapping and sandpile collapse problems.

search for, and escape across, the rare low barrier (4.3), in a frozen landscape of large $\phi$ barriers of typical height $\gamma$.

At a given temperature $\varepsilon$, sandpile collapse clearly depends on the nature of the density fluctuations. We look at two opposite cases of non-Gaussianness. If density fluctuations are peaked around zero (i.e., the material is almost non-dilatant), this implies a much flatter, more ordered $\phi$-configurational landscape, easier for the exciton to traverse. This would lead to a 'liquid-like' scenario of frequent collapse, where a finite angle of repose would be hard to sustain under any circumstances. An explicit example is provided by the $\gamma \rightarrow 0$ limit, where the collapse probability scales as $\varepsilon^{1 / 4}$ [see (4.2)].

In the opposite case of strong dilatancy (where large values of $\phi$ are more frequent than in the Gaussian equilibrium distribution), sandpile collapse is even more strongly inhibited. If, for example, $|\phi(t)|$ is constrained to remain larger than some threshold $\phi_{\text {th }}$, the stretched exponential in (4.3) reverts (in the $\varepsilon \ll 1$ regime considered) to an Arrhenius law in its usual form:

$$
\Pi \sim \exp \left(-\frac{\left(\phi_{\mathrm{th}} / \phi_{\mathrm{eq}}\right)^{4}}{2 \varepsilon}\right) .
$$

This would arise in the case of a strongly dilatant material, such as wet sand; angles of repose for such materials can be far steeper than usual, and still resist collapse.

\section{Discussion}

In the above, we have looked at a very familiar problem, that of the decay and eventual collapse of the angle of repose, using an approach that combines new ideas with very traditional concepts such as dilatancy [11]. Our simple theory suggests that sandpiles created at arbitrarily large angles will decay quickly to the maximal angle of stability; their subsequent behaviour is bistable, with jamming at a typical finite angle of repose as one outcome, or a continuing fast decay to zero, as another. All of this occurs because of dynamical competition between the fast dynamics of angle decay and the slow dynamics of density fluctuations, especially in the low-noise regime. The collapse of a sandpile in the jammed regime is shown to be a rare event; we have obtained exact results for the 
ensuing activated process, which turns out to have interesting analogies with the wellknown trapping problem. Using these analogies, we are able to summarise the process of collapse as follows: weakly dilatant sandpiles collapse easily, while strongly dilatant ones bounce back.

\section{Acknowledgments}

AM warmly thanks SMC-INFM (Research and Development Center for Statistical Mechanics and Complexity, Rome, Italy), and the Service de Physique Théorique, CEA Saclay, where parts of this work were done. Kirone Mallick is gratefully acknowledged for interesting discussions, and for having drawn our attention to Reference [16] after the present investigation was completed.

\section{Appendix. Equilibrium probability distribution for the process (2.1), (2.2)}

In this Appendix we derive an exact expression for the double Laplace transform of the joint equilibrium probability distribution $P(\theta, \phi)$ of the variables $\theta$ and $\phi$, for arbitrary values of the dimensionless parameters $\varepsilon$ and $\gamma$, and especially in the regime (2.5).

Our starting point consists in writing down the stationary (i.e., time-independent) Fokker-Planck equation describing the equilibrium state of the two-dimensional Markov process (2.1), (2.2). This equation reads [15]

$$
\frac{\partial J_{\theta}}{\partial \theta}+\frac{\partial J_{\phi}}{\partial \phi}=0
$$

where

$$
J_{\theta}=-\left(a \theta-b \phi^{2}\right) P-\Delta_{1}^{2} \frac{\partial P}{\partial \theta}, \quad J_{\phi}=-c \phi P-\Delta_{2}^{2} \frac{\partial P}{\partial \phi},
$$

i.e.,

$$
\Delta_{1}^{2} \frac{\partial^{2} P}{\partial \theta^{2}}+\Delta_{2}^{2} \frac{\partial^{2} P}{\partial \phi^{2}}+\left(a \theta-b \phi^{2}\right) \frac{\partial P}{\partial \theta}+c \phi \frac{\partial P}{\partial \phi}+(a+c) P=0 .
$$

We introduce the dimensionless variables

$$
\widehat{\theta}=\frac{\theta}{\theta_{\mathrm{r}}}, \quad \widehat{\phi}=\frac{\phi}{\phi_{\mathrm{eq}}},
$$

where

$$
\phi_{\mathrm{eq}}^{2}=\frac{\Delta_{2}^{2}}{c}, \quad \theta_{\mathrm{r}}=\frac{b \Delta_{2}^{2}}{a c}
$$

have been introduced in (3.4) and (3.6), and define the double Laplace transform of $P(\theta, \phi)$ as

$$
L(x, y)=\langle\exp (-x \widehat{\theta}-y \widehat{\phi})\rangle=\iint \exp (-x \widehat{\theta}-y \widehat{\phi}) P(\theta, \phi) \mathrm{d} \theta \mathrm{d} \phi .
$$

In terms of this function, the Fokker-Planck equation (A.3) becomes

$$
x\left(\frac{\partial^{2} L}{\partial y^{2}}+\frac{\partial L}{\partial x}\right)=\varepsilon x^{2} L+\gamma y\left(y L-\frac{\partial L}{\partial y}\right),
$$


together with the normalisation $L(0,0)=1$. The dimensionless parameters

$$
\gamma=\frac{c}{a}, \quad \varepsilon=\frac{a c^{2} \Delta_{1}^{2}}{b^{2} \Delta_{2}^{4}},
$$

have been introduced in (2.6) .

Our main interest will reside in the distribution of $\hat{\theta}$, encoded in the Laplace transform

$$
f(x)=L(x, 0)=\langle\exp (-x \widehat{\theta})\rangle
$$

so that

$$
P(\widehat{\theta})=\int \frac{\mathrm{d} x}{2 \mathrm{i} \pi} \mathrm{e}^{x \widehat{\theta}} f(x)
$$

and especially in the probability for $\theta$ to be negative:

$$
\Pi=\operatorname{Prob}(\theta<0)=\int_{-\infty}^{0} P(\widehat{\theta}) \mathrm{d} \widehat{\theta}=\int \frac{\mathrm{d} x}{2 \mathrm{i} \pi} \frac{f(x)}{x} .
$$

We first notice that

$$
L(0, y)=\mathrm{e}^{y^{2} / 2}
$$

obeys (A.7), in agreement with the plain observation that the stationary distribution of $\widehat{\phi}$ is a Gaussian with unit variance. The full problem can be solved by making the Ansatz that $L(x, y)$ keeps the same functional form in $y$ for any fixed value of $x$, i.e., looking for a solution to (A.7) of the form

$$
L(x, y)=f(x) \exp \left(g(x) \frac{y^{2}}{2}\right)
$$

with $f(0)=g(0)=1$, where $g(x)$ is the ' $x$-dependent variance' of $\phi$. Equation (A.7) boils down to two ordinary differential equations for $f(x)$ and $g(x)$ :

$$
\begin{aligned}
& x\left(g^{\prime}+2 g^{2}\right)+2 \gamma(g-1)=0, \\
& f^{\prime}=(\varepsilon x-g) f,
\end{aligned}
$$

justifying thus the validity of the above Ansatz.

The above equations can be solved as follows. Equation (A.14 is a Riccati equation [19] for $g(x)$, which can be linearised by setting

$$
g=\frac{1}{2}\left(\frac{\psi^{\prime}}{\psi}-\frac{\gamma}{x}\right) \text {. }
$$

The new unknown function $\psi(x)$ obeys the second-order linear equation

$$
\psi^{\prime \prime}=\left(\frac{\gamma(\gamma-1)}{x^{2}}+\frac{4}{x}\right) \psi
$$

whose normalised regular solution reads

$$
\psi(x)=x^{\gamma} \sum_{n \geq 0} \frac{\Gamma(2 \gamma)}{\Gamma(n+2 \gamma)} \frac{(4 \gamma x)^{n}}{n !}=\frac{\Gamma(2 \gamma)}{(4 \gamma)^{\gamma}} 2 \sqrt{\gamma x} I_{2 \gamma-1}(4 \sqrt{\gamma x})
$$


where $I_{2 \gamma-1}$ is the modified Bessel function. Equations (A.16) and A.15) then respectively yield the explicit expressions

$$
g(x)=\sqrt{\frac{\gamma}{x}} \frac{I_{2 \gamma}(4 \sqrt{\gamma x})}{I_{2 \gamma-1}(4 \sqrt{\gamma x})}
$$

and

$$
f(x)=f_{0}(x) \mathrm{e}^{\varepsilon x^{2} / 2},
$$

with

$$
\begin{aligned}
f_{0}(x)=\left(\frac{x^{\gamma}}{\psi(x)}\right)^{1 / 2} & =\left(\sum_{n \geq 0} \frac{\Gamma(2 \gamma)}{\Gamma(n+2 \gamma)} \frac{(4 \gamma x)^{n}}{n !}\right)^{-1 / 2} \\
& =\left(\frac{(4 \gamma x)^{\gamma-1 / 2}}{\Gamma(2 \gamma) I_{2 \gamma-1}(4 \sqrt{\gamma x})}\right)^{1 / 2} .
\end{aligned}
$$

The product formula (A.20) for $f(x)$ expresses that the variable $\hat{\theta}$ is the convolution of two independent random variables:

(i) a positive random variable $\widehat{\theta}_{0}$ whose distribution, encoded in $f_{0}(x)$, only depends on $\gamma$;

(ii) a Gaussian variable with variance $\varepsilon$.

The cumulants $c_{n}$ of $\widehat{\theta}$ can be obtained by means of the series expansion

$$
\sum_{n \geq 1} \frac{c_{n}(-x)^{n}}{n !}=\ln f(x)=\frac{\varepsilon x^{2}}{2}-\frac{1}{2} \ln \sum_{n \geq 0} \frac{\Gamma(2 \gamma)}{\Gamma(n+2 \gamma)} \frac{(4 \gamma x)^{n}}{n !} .
$$

We thus get

$$
\begin{aligned}
& c_{1}=1, \quad c_{2}=\frac{2}{2 \gamma+1}+\varepsilon, \quad c_{3}=\frac{8}{(\gamma+1)(2 \gamma+1)}, \\
& c_{4}=\frac{48(5 \gamma+3)}{(\gamma+1)(2 \gamma+1)^{2}(2 \gamma+3)}, \quad c_{5}=\frac{384(7 \gamma+6)}{(\gamma+1)(\gamma+2)(2 \gamma+1)^{2}(2 \gamma+3)}, \\
& c_{6}=\frac{3840\left(42 \gamma^{3}+118 \gamma^{2}+107 \gamma+30\right)}{(\gamma+1)^{2}(\gamma+2)(2 \gamma+1)^{3}(2 \gamma+3)(2 \gamma+5)},
\end{aligned}
$$

and so on. All the cumulants of $\widehat{\theta}$ coincide with those of $\widehat{\theta}_{0}$, except $c_{2}$, whose term linear in $\varepsilon$ represents the Gaussian variable in (ii) above.

Let us first consider the limiting situation $\gamma=0$, which is reached when the rate $c$ vanishes, so that the dynamics of the variable $\phi$ is entirely frozen. The above solution simplifies greatly. Indeed only the terms corresponding to $n=0$ and $n=1$ survive in the series representation (A.18) for $\psi(x)$, so that

$$
\psi(x)=1+2 x,
$$

and therefore

$$
f_{0}(x)=(1+2 x)^{-1 / 2}, \quad g(x)=\frac{1}{1+2 x} .
$$

The expression for $f_{0}(x)$ shows that $\widehat{\theta}_{0}$ is nothing but the square of a normalised Gaussian variable, the latter being identified with $\widehat{\phi}$. 
The probability $\Pi$ for $\theta$ to be negative therefore reads

$$
\Pi=\int \frac{\mathrm{d} x}{2 \mathrm{i} \pi} \frac{\mathrm{e}^{\varepsilon x^{2} / 2}}{x \sqrt{1+2 x}} .
$$

In the regime $\varepsilon \ll 1$ of most interest, this expression simplifies as

$$
\Pi \approx \int \frac{\mathrm{d} x}{2 \mathrm{i} \pi} \frac{\mathrm{e}^{\varepsilon x^{2} / 2}}{\sqrt{2 x^{3}}} \approx \frac{(2 \varepsilon)^{1 / 4}}{\Gamma(1 / 4)} .
$$

In the general situation where $\varepsilon$ and $\gamma$ are both non-zero, the results (A.19)-A.21) are much more involved. The following situations deserve our attention:

- For $\varepsilon=0$, i.e., in the absence of noise in (2.1), the variable $\hat{\theta}$ reduces to $\widehat{\theta}_{0}$. Its probability density reads

$$
P_{0}(\widehat{\theta})=\int \frac{\mathrm{d} x}{2 \mathrm{i} \pi} \mathrm{e}^{x \widehat{\theta}}\left(\frac{(4 \gamma x)^{\gamma-1 / 2}}{\Gamma(2 \gamma) I_{2 \gamma-1}(4 \sqrt{\gamma x})}\right)^{1 / 2} .
$$

The parenthesis falls off exponentially for $x \rightarrow+\infty$, confirming thus that $\widehat{\theta}$ is positive. As $\hat{\theta} \rightarrow 0$, the integral in the right-hand side is dominated by large values of $x$, where the Bessel function can be approximated by its asymptotic expression

$$
I_{2 \gamma-1}(z) \approx \frac{e^{z}}{\sqrt{2 \pi z}}
$$

irrespective of $\gamma$. A saddle-point approximation yields the estimate

$$
P_{0}(\widehat{\theta}) \approx \frac{(2 \gamma / \hat{\theta})^{\gamma}}{\Gamma(2 \gamma+1)^{1 / 2}}\left(\frac{8 \gamma^{3}}{\pi \widehat{\theta}^{5}}\right)^{1 / 4} \exp \left(-\frac{\gamma}{\hat{\theta}}\right) .
$$

The probability density of $\widehat{\theta}$ therefore falls off exponentially fast for $\widehat{\theta} \ll \gamma$.

- For $\varepsilon>0$, the probability for $\widehat{\theta}$ to be negative,

$$
\Pi=\int \frac{\mathrm{d} x}{2 \mathrm{i} \pi x} \mathrm{e}^{\varepsilon x^{2} / 2}\left(\frac{(4 \gamma x)^{\gamma-1 / 2}}{\Gamma(2 \gamma) I_{2 \gamma-1}(4 \sqrt{\gamma x})}\right)^{1 / 2},
$$

is non-zero. As $\varepsilon \rightarrow 0$, the integral in the right-hand side is again dominated by large values of $x$, and a saddle-point approximation yields the estimate

$$
\Pi \approx \frac{\left(8 \gamma^{2} / \varepsilon\right)^{\gamma / 3}}{\Gamma(2 \gamma+1)^{1 / 2}}\left(\frac{8 \varepsilon}{9 \pi}\right)^{1 / 4} \exp \left(-\frac{3}{2}\left(\frac{\gamma^{2}}{\varepsilon}\right)^{1 / 3}\right) .
$$

The probability $\Pi$ therefore falls off as a stretched exponential for $\varepsilon \ll \gamma^{2}$.

- In the $\gamma \rightarrow 0$ limit, the results (A.30) and A.32 simplify, as the first factors of both expressions go to unity. Furthermore, throughout the regime (2.5), i.e., whenever $\varepsilon$ and $\gamma$ are simultaneously small, the probability $\Pi$ obeys a scaling law of the form

$$
\Pi \approx \frac{(2 \varepsilon)^{1 / 4}}{\Gamma(1 / 4)} \mathcal{F}(\zeta), \quad \zeta=\frac{\gamma}{\varepsilon^{1 / 2}} .
$$

This result interpolates between (A.27) and A.32). In order to derive the expression of the scaling function $\mathcal{F}(\zeta)$, it is again convenient to come back to the series 
representation (A.18) for $\psi(x)$. For $\gamma \ll 1$, keeping $z=4 \sqrt{\gamma x}$ finite, that expression simplifies as

$$
\psi(x) \approx \frac{z I_{1}(z)}{4 \gamma}
$$

Inserting this scaling estimate into (A.20) and (A.11), we obtain after some algebra

$$
\mathcal{F}(\zeta)=2^{7 / 4} \Gamma(1 / 4) \zeta^{1 / 2} \int_{\mathrm{e}^{-\mathrm{i} \pi / 4} \infty}^{\mathrm{e}^{\mathrm{i} \pi / 4} \infty} \frac{\mathrm{d} z}{2 \mathrm{i} \pi}\left(z^{3} I_{1}(z)\right)^{-1 / 2} \exp \left(\frac{z^{4}}{512 \zeta^{2}}\right) .
$$

This is a monotonically decreasing function of $\zeta$, starting from the value $\mathcal{F}(0)=1$, so that (A.27) is recovered. Its fall-off at large values of $\zeta$,

$$
\mathcal{F}(\zeta) \approx \Gamma(1 / 4)\left(\frac{4}{9 \pi}\right)^{1 / 4} \exp \left(-\frac{3}{2} \zeta^{2 / 3}\right)
$$

agrees with (A.32) in the $\gamma \rightarrow 0$ limit.

\section{References}

[1] Brown R L and Richards J C 1966 Principles of Powder Mechanics (Oxford: Pergamon)

[2] Bak P, Tang C, and Wiesenfeld K 1987 Phys. Rev. Lett. 59381

[3] Nagel S R 1992 Rev. Mod. Phys. 64321

[4] Mehta A, Needs R J, and Dattagupta S 1992 J. Stat. Phys. 681131

[5] Bagnold R A 1966 Proc. R. Soc. London Ser. A 295219

[6] Mehta A and Barker G C 2001 Europhys. Lett. 56626

[7] Daerr A and Douady S 1999 Nature 399241

[8] Jaeger H M, Liu C H, and Nagel S R 1989 Phys. Rev. Lett. 6240

[9] Luck J M and Mehta A 2003 J. Phys. A 36 L365

2003 Eur. Phys. J. B 35399

[10] Edwards S F 1998 Physica 249226

[11] Reynolds O 1885 Phil. Mag. 20469

[12] Berg J and Mehta A 2001 Europhys. Lett. 56784

[13] Mehta A, Barker G C, and Luck J M 2003 preprint cond-mat/0312410

[14] Uhlenbeck G E and Ornstein L S 1930 Phys. Rev. 36823

Wang M C and Uhlenbeck G E 1945 Rev. Mod. Phys. 17323

[15] van Kampen N G 1992 Stochastic Processes in Physics and Chemistry (Amsterdam: NorthHolland)

[16] Luczka J 1986 J. Stat. Phys. 421009

1986 J. Stat. Phys. 45309

1987 J. Stat. Phys. 47505

[17] Edwards S F 1994 in Granular Matter: An Interdisciplinary Approach edited by Mehta A (NewYork: Springer)

[18] Smoluchowski M V 1916 Phys. Z. 17557

Donsker M D and Varadhan S R S 1975 Commun. Pure Appl. Math. 28525

1979 Commun. Pure Appl. Math. 32721

Grassberger P and Procaccia I 1982 J. Chem. Phys. 776281

Anlauf J K 1984 Phys. Rev. Lett. 521845

Haus J W and Kehr K W 1987 Phys. Rep. 150263

Luck J M 1992 Systèmes désordonnés unidimensionnels (in French) (Saclay: Collection Aléa)

[19] Zwillinger D 1992 Handbook of Differential Equations 2nd ed (New-York: Academic) 